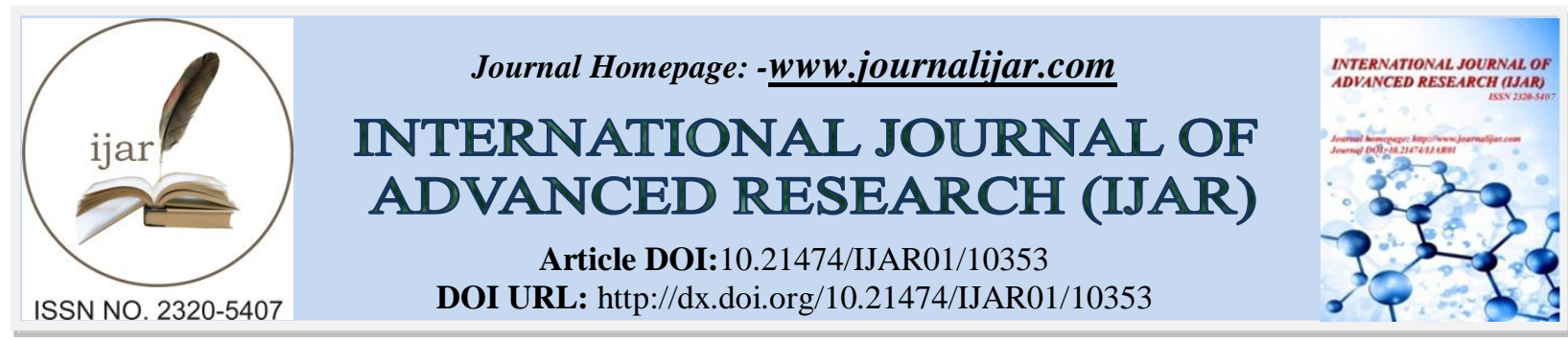

RESEARCH ARTICLE

\title{
LESIONS ERYTHEMATO - SQUAMEUSES: PENSER A LA POROKERATOSEERYTHEMATO SQUAMOUS LESIONS : THINKING TO POROKERATOSIS
}

\author{
Soundous Ben Moussa ${ }^{1}$, Meriem Abid ${ }^{1}$, Sofia Berrada ${ }^{2}$, Fatim-Zahra Hazmiri ${ }^{2}$, Ouafa Hocar ${ }^{1}$, Hanane Raiss $^{2}$ \\ and Said Amal ${ }^{1}$ \\ 1. Service De Dermatologie Et De Vénérologie, Hôpital Arrazi, CHU Mohammed VI, Marrakech. \\ 2. Service D'anatomie Pathologique, Hôpital Arrazi, CHU Mohammed VI, Marrakech.
}

\section{Manuscript Info}

Manuscript History

Received: 27 November 2019

Final Accepted: 30 December 2019

Published: January 2020

Key words:-

Erythemato-Squamous Lesions, Linear

Porokératosis, Child

\section{Abstract}

Copy Right, IJAR, 2020,. All rights reserved.

\section{Introduction:-}

La porokératose est une dermatose peu fréquente caractérisée par un trouble de la différenciation épidermique(Truchetet et Cuny, 2017). L'étiopathogénie n'est pas encore entièrement élucidée, mais parait complexe et multifactorielle.

\section{Observation:-}

Nous rapportons le cas d'une enfant âgée de 7ans admise en consultation pour des lésions érythémato-squameuses trainantes depuis 2 ans. L'examen dermatologique avait objectivé des lésions papuleuses érythémato-squameuses confluentes par endroits, formant des plaques de taille et de forme variable à contours nets, localisées au niveau de la face dorsale de la main gauche et au niveau de la face postérieure du coude gauche ( figure1). L'examen des muqueuses et des phanères était normal. Le reste de l'examen clinique était sans particularités. Nous avons réalisé une biopsie cutanée qui a objectivé une image de lamelle cornoïde concluant à une porokératose (figure 2). L'enfant a été traitée par voie locale à base de trétinoine et photoprotection avec une bonne amélioration.

\section{Discussion:-}

La porokératose est une entité rare décrite pour la première fois en 1893 par Vittorio Mibelli (Mibelli et al , 1983). Il existe plusieurs formes anatomo-cliniques dont la plus fréquente est la porokératose en plaques de Mibelli. Dans notre cas, il s'agissait d'une forme linéaire qui est une variante rare caractérisée par de petites papules kératosiques brunâtres qui se développent lentement depuis l'enfance pour former des plaques annulaires irrégulières avec des bordures surélevées bien délimitées et un centre atrophique. Elle sont strictement unilatérales suivant les lignes de blaschko(Ruiz Villaverde et al ,2005). L'aspect histologique caractéristique objective l'image de la lamelle cornoide: la couche granuleuse comporte une interruption focale nette surmontée d'un long empilement de cellules cornées parakératosiques avec des kératinocytes dysplasiques du stratum spinosum sous-jacent. L'évolution se fait vers une aggravation lente mais progressive. Des régressions spontanées, après une poussée inflammatoire, sont possibles mais restent rares (Kanzaki et al, 1992). Les lésions peuvent subir une transformation maligne en carcinome spinocellulaire, maladie de Bowen, plus rarement carcinome basocellulaire ou mélanome (Maubec et al, 
2005). Les traitements sont justifiés par le risque de transformation maligne et par l'aspect inesthétique des lésions sur des zones visibles. Les différentes méthodes interventionnelles proposées sont la chirurgie, la cryothérapie, la photovolatisation au laser (CO2,Q-switched, ruby), la dermabrasion, la photothérapie dynamique. Des traitements locaux avec ou sans occlusion ont été proposés (émollients, kératolytiques, 5-fluoro-uracile, dithranol, trétinoïne, calcipotriol, dermocorticoïdes, diclofénac, imiquimod $5 \%$, mébutate d'ingénol) (Truchetet et Cuny, 2017).

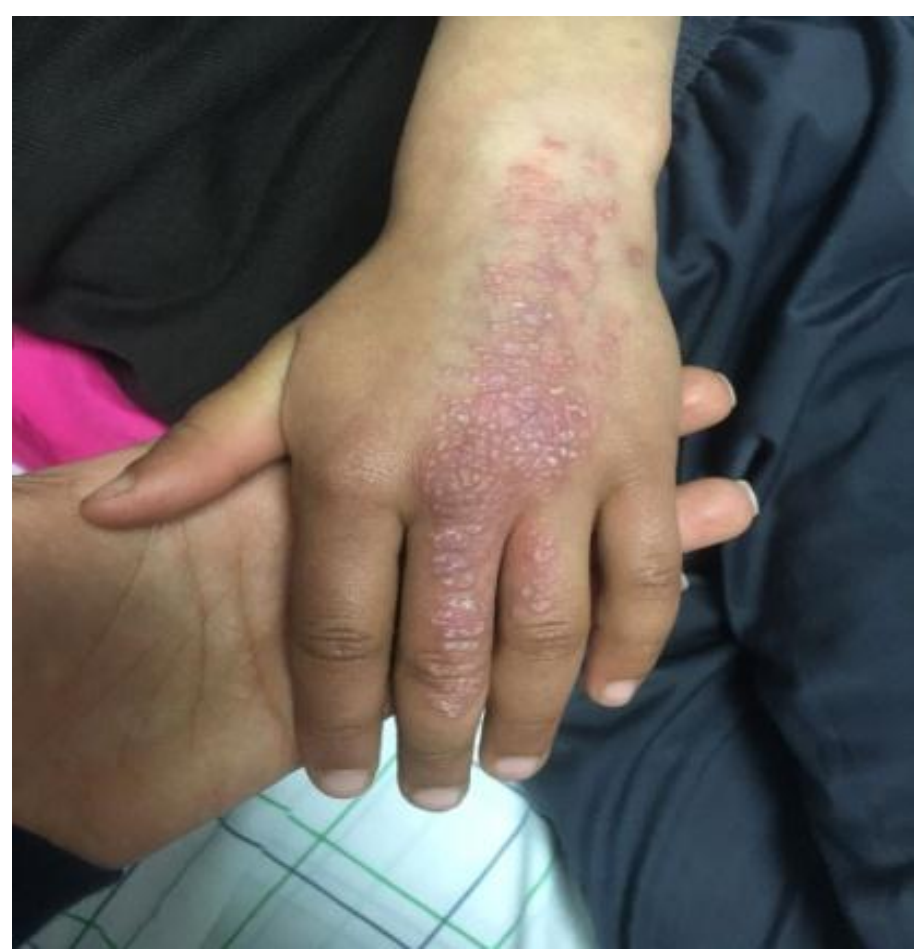

figure 1:- Lésions de porokératose chez notre patiente.

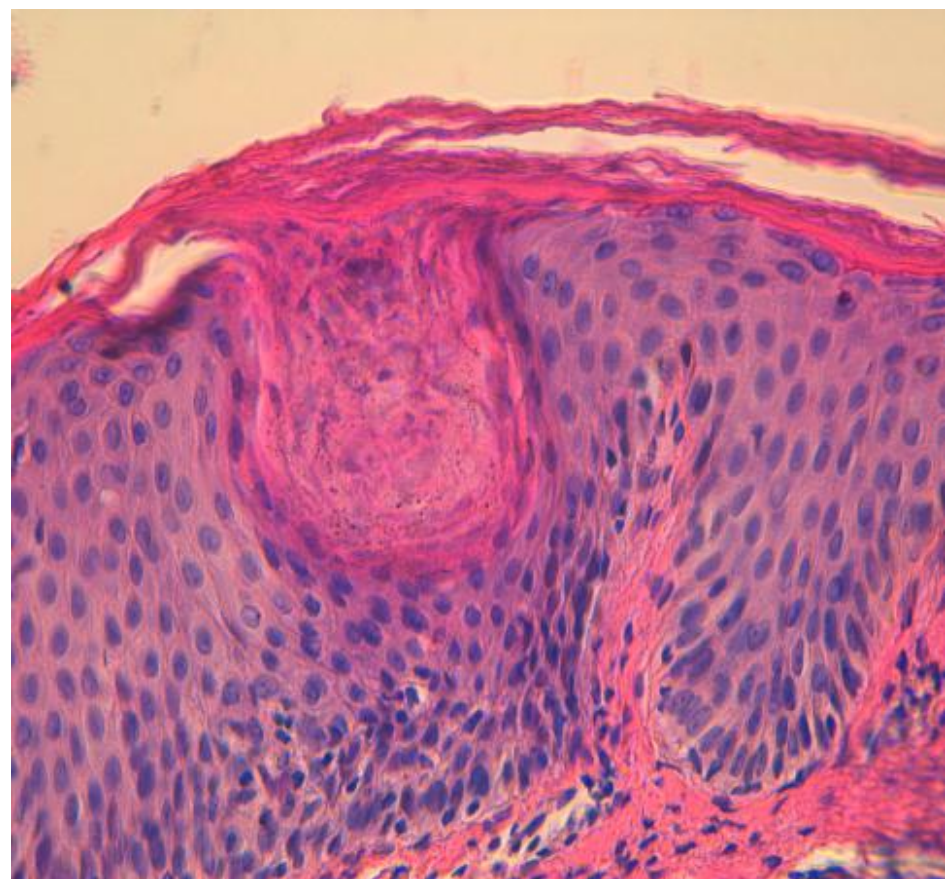

figure 2:- aspect histologique de la lamelle cornoïde. 


\section{Conclusion:-}

La porokératose est un trouble rare de kératinisation. Son pronostic global est favorable, toutefois, conditionné par le risque de transformation maligne des lésions, d'où l'importance d'une surveillance régulière.

\section{Références:-}

1. Kanzaki T, Miwa N, Kobayashi T, Ogawa S. Eruptive pruritic papular porokeratosis. J Dermatol 1992;19:109_ 12

2. Maubec E, Duvillard P, Margulis A, Bachollet B, Degois G, Avril MF. Common skin cancers in porokeratosis. Br J Dermatol 2005;152:1389-91.

3. Mibelli V. Contributo allo etudio Della ipercheratosi deicanali sudoriferi (porokeratosis). G Ital Mal Veneree Pelle.1893;28:313-35. Cited in, McDonald SG, Peterka ES. Treatment with topical 5-fluorouracil. J Am Acad Dermatol. 1983;8:107-10

4. Ruiz Villaverde R, Alonso Corral MJ, Sánchez Cano D, Pacheco Sa'nchez-Lafuente FJ. Linear porokeratosis of Mibelli [in Spanish]. An Pediatr (Barc). 2005;63 (4):376-377

5. F. Truchetet, J.-F. Cuny, Dermatologie et infections sexuellement transmissibles, p363, (c) 2017, Elsevier Masson SAS. 